\title{
The presentation, management and outcomes of Fournier's gangrene at a tertiary urology referral centre in South Africa
}

\author{
A E Elsaket, ${ }^{1,2} \mathrm{MB}$ BCh, FC Urol (SA); S Maharajh, ${ }^{3} \mathrm{MB}$ ChB, FCUrol (SA), MMed (Urol); \\ R J Urry, ${ }^{1,2}$ BSc Hons, MB BCh, FC Urol (SA), MMed (Urol) \\ ${ }^{1}$ Department of Urology, Grey's Hospital, Pietermaritzburg, South Africa \\ ${ }^{2}$ School of Clinical Medicine, Nelson R Mandela School of Medicine, College of Health Sciences, University of KwaZulu-Natal, Durban, South Africa \\ ${ }^{3}$ Department of Urology, Inkosi Albert Luthuli Central Hospital, Durban, South Africa
}

Corresponding author: R J Urry (james@urry.co.za)

\begin{abstract}
Background. Fournier's gangrene (FG) is a clinically relevant condition with a high mortality rate. In South Africa (SA) most affected patients present at district and regional level hospitals. It is important for doctors to recognise the condition and accurately assess patients with FG to decide which of them need urgent referral to a tertiary centre.

Objectives. To review the presentation, management and outcomes of patients with FG at a tertiary urology referral centre, with the specific intention of identifying prognostic factors and assessing the validity of the Fournier's Gangrene Severity Index (FGSI).

Methods. A retrospective chart review was performed of all patients treated for FG over a 5-year period at Grey's Hospital in Pietermaritzburg, SA. HIV-positive patients were compared with patients with diabetes mellitus (DM). The FGSI was calculated for each patient. Regression analysis was performed to identify risk factors.

Results. Forty-four patients (mean age 51 years) were treated for FG, corresponding to 8.8 patients per year. HIV was the commonest comorbidity, followed by DM. HIV-positive patients presented at a younger age than non-HIV-positive patients $(p<0.001)$. On average the patients underwent 1.33 debridements, and $45.5 \%$ required transfusion. All were treated with broad-spectrum antibiotics. The overall mortality rate was $11.4 \%$ and the mean hospital length of stay was 26 days. There was no difference between the mean age of survivors and non-survivors $(p=0.752)$. There was no association between mortality, HIV, DM or number of debridements. The mean (standard deviation) FGSI was significantly different in patients who died $(15.4(4.78))$ and those who survived $(5.92(4.09))(p<0.001)$. There was a significant association between FGSI $>9$ and mortality $(p=0.017)$. FGSI $>9$ predicted $44.4 \%$ mortality, and FGSI $\leq 9$ predicted $95.5 \%$ survival. A combination of FGSI $>9$, debridement outside the perineum (onto the abdominal wall, chest or limbs) and requirement for organ support was present in $80.0 \%$ of patients who died and was a significant risk factor for mortality ( $p=0.002)$.

Conclusions. In a resource-constrained environment such as SA, outcome prediction is necessary to enable resource allocation. Patients with an FGSI $>9$ have a high risk of mortality and will benefit from ICU care. The combination of FGSI $>9$, requirement for organ support and extension beyond the perineum is associated with a very high risk of mortality and may be useful as an exclusion criterion when allocating scarce resources.
\end{abstract}

S Afr Med J 2018;108(8):671-676. DOI:10.7196/SAMJ.2018.v108i8.13100

Fournier's gangrene (FG) is a clinically relevant condition in South Africa (SA), particularly at regional and district-level hospitals, where most affected patients present. Although relatively rare, it has a high mortality rate of up to $30 \%{ }^{[1]}$ Failure to make the correct diagnosis or delay in initiating definitive management can have a devastating impact on the chance of survival. It is important for doctors to recognise the condition and have the necessary clinical tools to accurately assess patients with FG and decide which of them need urgent referral to a tertiary centre.

FG is a polymicrobial infection of the perineum, scrotum and penis that spreads in fascial planes and causes obliterative endarteritis of subcutaneous arteries, resulting in gangrene of the overlying tissue and skin. ${ }^{[2]}$ Patients with FG usually have underlying impaired immunity as is seen in diabetes mellitus (DM), HIV infection and AIDS, chronic alcoholism, liver disease, renal disease and immune suppression after a transplant. ${ }^{[3,4]} \mathrm{SA}$ has the highest prevalence of HIV in the world, ${ }^{[5]}$ and a positive correlation between HIV infection and FG has already been established. ${ }^{[6]}$

FG is most commonly caused by polymicrobial infection of aerobes and anaerobes. These usually include Escherichia coli, Streptococcus spp., Staphylococcus spp., Clostridium spp., Bacteroides spp. and Corynebacterium spp ${ }^{[1]}$ Other rare causes such as fungal infection ${ }^{[7]}$ and atypical bacteria ${ }^{[8]}$ have been described. The organisms may be skin flora, which act synergistically and invade and spread in the subcutaneous tissue because of the immunocompromised status of the host. There may also be an initiating event such as a periurethral abscess, urethral stricture, skin abscess or perianal abscess. In hospitalised patients, hospital-acquired methicillin-resistant $S$. aureus may cause FG. ${ }^{[9]}$ FG generally affects men but can affect women, and it can occur at any age.

The necrotising fasciitis of FG involves the superficial and deep fascial planes of the perineum. The overlying skin may be involved, but the muscular structures deep to the fascia and the testes are usually spared because they have an independent blood supply. Scarpa's fascia is continuous with Colles fascia (the superficial perineal fascia), and Dartos fascia of the penis and scrotum. This fascial plane is in continuity from the perineum to the clavicles. Infection of any of the perineal structures can progress into the superficial perineal space and access the fascial planes, with the potential to spread and involve the perineum, scrotum and penis and the abdominal wall up to the 
clavicles. ${ }^{[10]}$ Fig. 1 shows a patient with FG of the penis, scrotum and perineum at the time of debridement in theatre.

Management of FG is aggressive and multimodal, consisting of resuscitation, broad-spectrum antibiotics and surgical debridement. ${ }^{[1,3]}$ Diversion of urine and stool may be necessary. Some patients may require intensive care unit (ICU) admission. Furthermore, repeated debridement ${ }^{[11]}$ and specialised wound care are necessary in some patients. The Fournier's Gangrene Severity Index (FGSI ${ }^{[2]}$ is one of several assessment tools that can be used to assess the severity of FG.

\section{Objectives}

To review the management and outcomes of FG, compare outcomes in patients with DM and HIV-positive patients, identify prognostic factors that may contribute to decision-making in the management of FG, and assess the validity of the FGSI in SA patients.

\section{Methods}

Grey's Hospital is a tertiary 530-bed academic hospital in Pietermaritzburg, SA. It has a wide catchment area covering a population of $\sim 3$ million people. It is the tertiary referral centre for urology for

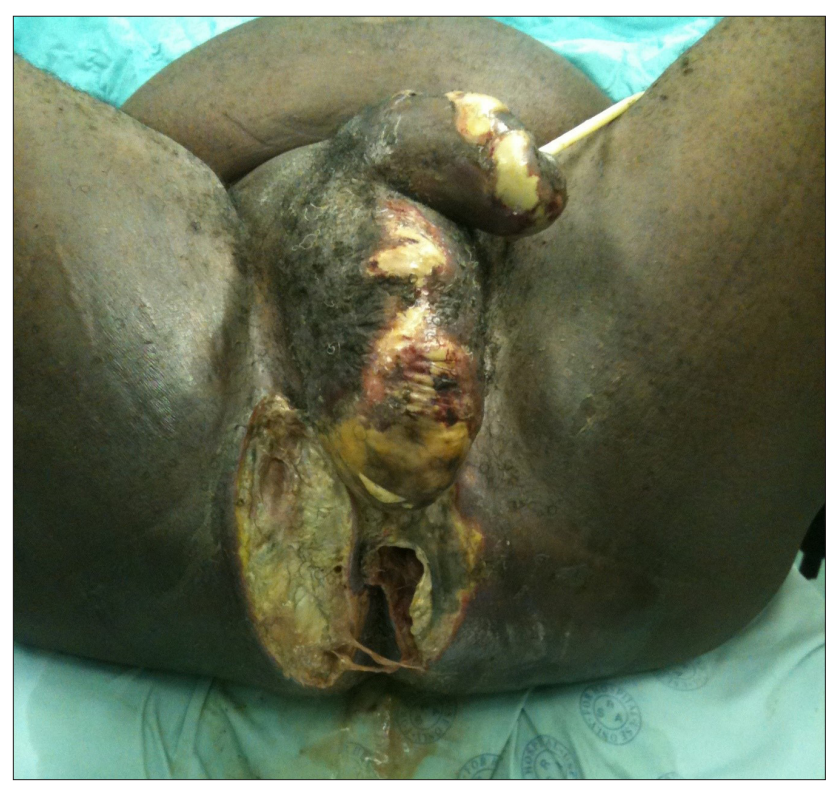

Fig. 1. Fournier's gangrene involving the penis, scrotum and perianal area. the entire referral area, which includes primary healthcare clinics and district and regional hospitals. All patients treated for FG in the Department of Urology at Grey's Hospital between 1 January 2010 and 31 December 2014 were included in the study. A retrospective chart review was performed. Patient records were traced from the emergency department, ward, theatre and ICU. Parameters recorded included age, gender, comorbidities, metabolic parameters, serum haematological parameters, serum biochemical parameters, extent of debridement, number of re-debridements, organisms cultured, ICU admission, management, length of hospital stay and outcome. Diagnosis was made on the basis of history and clinical examination.

The FGSI ${ }^{[1]}$ was calculated for each patient using Microsoft Excel 365, 2016 release (Microsoft, USA). The index was automatically calculated on insertion of the relevant parameters. Table 1 outlines the parameters used to calculate the FGSI.

In the original study in which the FGSI was described in patients from New York, USA, patients with an FGSI of $>9$ had a $75 \%$ probability of death. Those with an FGSI of $\leq 9$ had a $78 \%$ probability of survival. ${ }^{[2]}$ In the present study, a combination of FGSI >9, debridement beyond the perineum and requirement for organ support were assessed together as a predictor for mortality. These were chosen because they are highly clinically relevant and suitable to calculate quickly at the bedside.

Ethics approval for the study was granted by the Biomedical Research Ethics Committee of the University of KwaZulu-Natal (ref. no. BCA237/15).

\section{Statistical analysis}

Data were collected and tabulated using Microsoft Excel 365, 2016 release (Microsoft, USA). Statistical analyses were performed using the Statistical Package for Social Sciences (SPSS) version 24 (IBM, USA). Comparison of means was performed using the $t$-test for equality of means in independent samples. Pearson's $\chi^{2}$ test was used to compare categorical variables. If the projected frequency, assuming a true null hypothesis, in a cell of a two-by-two table was less than five observations, Fisher's exact test was used. A $p$-value $<0.05$ (5\%) was considered statistically significant. Multiple univariate logistic regressions were performed to determine the odds ratio for risk factors for mortality. Following identification of risk factors, multivariate regression analysis was performed to confirm the significance of independent risk factors. Because of quasi-complete separation in the data resulting from the small sample size of patients who died, this was done using the Firth logistic regression.

\begin{tabular}{|c|c|c|c|c|c|c|c|c|c|}
\hline \multirow[b]{3}{*}{ Physiological variable } & \multicolumn{9}{|c|}{ Point assignment } \\
\hline & \multicolumn{4}{|c|}{ High abnormal values } & \multirow{2}{*}{$\begin{array}{c}\text { Normal } \\
(0)\end{array}$} & \multicolumn{4}{|c|}{ Low abnormal values } \\
\hline & +4 & +3 & +2 & +1 & & +1 & +2 & +3 & +4 \\
\hline Temperature $\left({ }^{\circ} \mathrm{C}\right)$ & $\geq 41$ & $39-40.9$ & - & $38.5-38.9$ & $36-38.4$ & $34-35.9$ & $32-33.9$ & $30-31.9$ & $\leq 29.9$ \\
\hline Heart rate (bpm) & $\geq 180$ & $140-179$ & $110-139$ & - & $70-109$ & - & $55-69$ & $40-54$ & $\leq 39$ \\
\hline Respiratory rate (/min) & $\geq 50$ & $35-49$ & - & $25-34$ & $12-24$ & $10-11$ & $6-9$ & - & $\leq 5$ \\
\hline Serum sodium $(\mathrm{mmol} / \mathrm{L})$ & $\geq 180$ & $160-179$ & $155-159$ & $150-154$ & $130-149$ & - & $120-129$ & $111-119$ & $\leq 110$ \\
\hline Serum potassium $(\mathrm{mmol} / \mathrm{L})$ & $\geq 7$ & $6-6.9$ & - & $5.5-5.9$ & $3.5-5.4$ & $3-3.4$ & $2.5-2.9$ & - & $<2.5$ \\
\hline $\begin{array}{l}\text { Serum creatinine ( } \mu \mathrm{mol} / \mathrm{L}) \text { (double } \\
\text { allocated score for acute renal failure) }\end{array}$ & $\geq 310$ & $177-309$ & $133-176$ & - & $53-175$ & - & $<53$ & - & - \\
\hline Haematocrit (\%) & $\geq 60$ & - & $50-59.9$ & $46-49.9$ & $30-45.9$ & - & $20-29.9$ & - & $<20$ \\
\hline White blood count $\left(\times 10^{9} / \mathrm{L}\right)$ & $\geq 40$ & - & $20-49.9$ & $15-19.9$ & $3-14.9$ & - & $1-2.9$ & - & $<1$ \\
\hline $\begin{array}{l}\text { Serum bicarbonate, venous }(\mathrm{mmol} / \mathrm{L}) \\
\text { bpm }=\text { beats per minute. }\end{array}$ & $\geq 52$ & $41-51.9$ & - & $32-40.9$ & $22-31.9$ & - & $18-21.9$ & $15-17.9$ & $<15$ \\
\hline
\end{tabular}




\section{Results}

During the 5-year study period, a total of 44 patients (8.8 patients per year) were diagnosed with and treated for FG. Of these, 43 were male and 1 was female. The mean age was 51 years (range 28 - 82).

\section{Comorbidities}

Fig. 2 illustrates the distribution of comorbid risk factors for FG. There were no patients on immunosuppressive medication.

The mean age of the HIV-positive patients was 41 years and that of the non-HIV-positive patients 60 years $(p<0.001)$. Similarly, the mean age of the DM patients was 62 years and that of the non-DM patients 43 years $(p<0.001)$.

\section{Initiating events}

Table 2 summarises the precipitating events leading to FG.

The two patients who developed FG after surgery initially underwent urethroplasty and inguinal hernia repair.

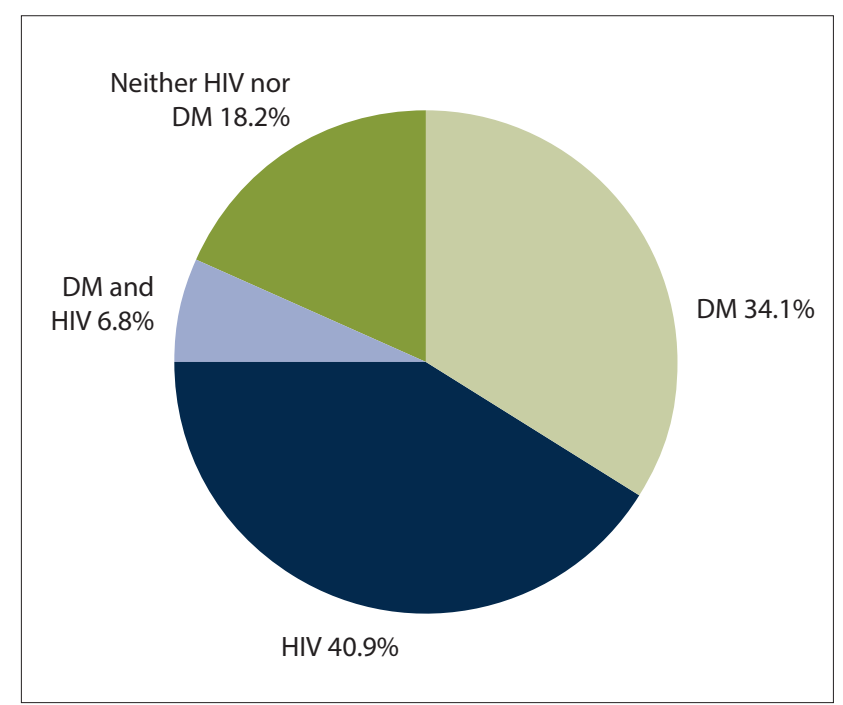

Fig. 2. Distribution of comorbid risk factors.

Table 2. Precipitating events leading to Fournier's gangrene $(N=44)$

\begin{tabular}{ll}
\hline Precipitating event & $\boldsymbol{n}(\%)$ \\
\hline Periurethral abscess or urethral stricture & $25(56.8)$ \\
Perianal source, e.g. perianal abscess & $10(22.7)$ \\
Spinal cord-injured patients, e.g. with septic bedsores & $3(6.8)$ \\
Idiopathic & $3(6.8)$ \\
Post-surgical & $2(4.5)$ \\
Scrotal source, e.g. scrotal abscess & $1(2.3)$
\end{tabular}

Table 3. Clinical parameters on presentation

\begin{tabular}{llll}
\hline Parameter & Mean & Median & Range \\
\hline $\begin{array}{l}\text { Mean arterial pressure } \\
(\mathrm{mmHg})\end{array}$ & 95.27 & 95.67 & $63-130$ \\
Heart rate $(\mathrm{bpm})$ & 97 & 96 & $72-120$ \\
Temperature $\left({ }^{\circ} \mathrm{C}\right)$ & 36.9 & 36.8 & $35.3-39.1$ \\
$\mathrm{pH}$ on arterial blood gas & 7.37 & 7.38 & $7.22-7.48$ \\
Lactate $(\mathrm{mmol} / \mathrm{L})$ & 2.15 & 2.1 & $1-4.2$ \\
Haemoglobin $(\mathrm{g} / \mathrm{dL})$ & 10.57 & 11.9 & $3.7-14.5$ \\
Creatinine $(\mu \mathrm{mol} / \mathrm{L})$ & 128.8 & 101 & $74-766$
\end{tabular}

\section{Presentation}

The clinical parameters on presentation are summarised in Table 3.

Only two patients presented with an initial mean arterial pressure $<70 \mathrm{mmHg}$. These two and three others went on to require inotrope support for septic shock during or after surgical management. Renal failure was present in $34.1 \%$ of patients, the majority of which (93.3\%) was due to acute kidney injury (AKI). Eight patients (18.2\%) were acidotic on presentation with a $\mathrm{pH}<7.35$.

\section{Causative organisms and antibiotic use}

In 33 patients (75.0\%), there was a positive culture on wound swab. The commonest cultured organism was S. aureus (39.4\%), followed by Acinetobacter spp. (33.3\%), S. pyogenes (24.2\%) and Proteus mirabilis (3.0\%). Most patients had evidence of urinary tract infection (79.6\%), with E. coli cultured in the urine in $63.6 \%$ of patients and P. mirabilis in $15.9 \%$. There was no concordance between culture findings on wound swab and in urine.

All patients were treated with broad-spectrum antibiotics, the majority $(72.7 \%)$ per the local protocol with amoxicillin and clavulanic acid (co-amoxiclav), metronidazole and gentamicin. The remainder (27.3\%) were treated with co-amoxiclav alone.

\section{Management}

All the patients were catheterised by suprapubic (84.1\%) or urethral (15.9\%) catheter placement.

All but one of the patients (97.7\%) underwent surgical debridement. The patient who did not undergo surgical debridement was HIVpositive and quadriplegic with infected bedsores. He presented 18 days after initial signs of infection, in septic shock. He was not taken to theatre based on a multidisciplinary team decision that he was too ill to survive extensive debridement.

At debridement, fasciitis involving the penis and scrotum was found in all patients. In $72.7 \%$ of patients, the fasciitis extended posterior to the perineal body and debridement of the perianal area was also required. In $31.8 \%$ of patients, the fasciitis had spread to the anterior abdominal wall and debridement of the inguinal areas and abdominal wall was required. Fasciitis extended to the chest wall in one patient and down the thighs in another.

The mean number of surgical procedures per patient was 1.33 (range 1 - 3), and $29.6 \%$ of patients required multiple debridements. The mean surgical time was 81 minutes (range $33-240$ ). Three patients (6.8\%) had a diverting colostomy performed at the discretion of the surgical team, specifically for the indication of involvement of the anal sphincter. Orchidectomy was performed in four patients (9.1\%).

Twenty patients (45.5\%) required blood transfusion. The mean number of units transfused in these patients was 2.7 (range 1 - 6).

Fig. 3 shows a patient with FG immediately after extensive debridement of the left iliac fossa, and Fig. 4 shows the same patient after wound care, prior to skin grafting.

\section{Outcomes}

The mean hospital length of stay (LoS) was 26 days (range 9 - 48).

The overall mortality rate was $11.4 \%$. Two of the five patients who died had DM, two were HIV-positive and one had both DM and HIV. All the patients who died had fasciitis extending beyond the perineum. The patients with fasciitis extending onto the thighs and to the chest wall both died. Patients who died did so on average within 7 days of admission to the tertiary centre.

Six patients (13.6\%) were identified as requiring ICU admission based on the need for organ support. Owing to severe constraints 
on ICU bed availability and according to local protocols, four of these patients were refused ICU admission on the basis of being too ill and ultimately died. Two were accepted to the ICU, of whom one survived and the other died. The mortality rate in patients requiring ICU admission for organ support was $83.3 \%$.

\section{Predictors of outcome}

There was no difference between the mean age of survivors and non-survivors $(p=0.752)$, and no association between mortality and comorbidity ( $p=0.566$ ) or number of debridements $(p=0.087$ ).

There were sufficient data to calculate the FGSI retrospectively in 31 patients. The median FGSI was 6 (range 0 - 20). The mean (standard deviation) FGSI differed significantly between patients who died (15.4 (4.78)) and those who survived (5.92 (4.09)) $(p<0.001)$. There was a significant association between FGSI $>9$ and mortality $(p=0.017)$. FGSI $>9$ predicted $44.4 \%$ mortality, and FGSI $\leq 9$ predicted $95.5 \%$ survival. A combination of FGSI $>9$,

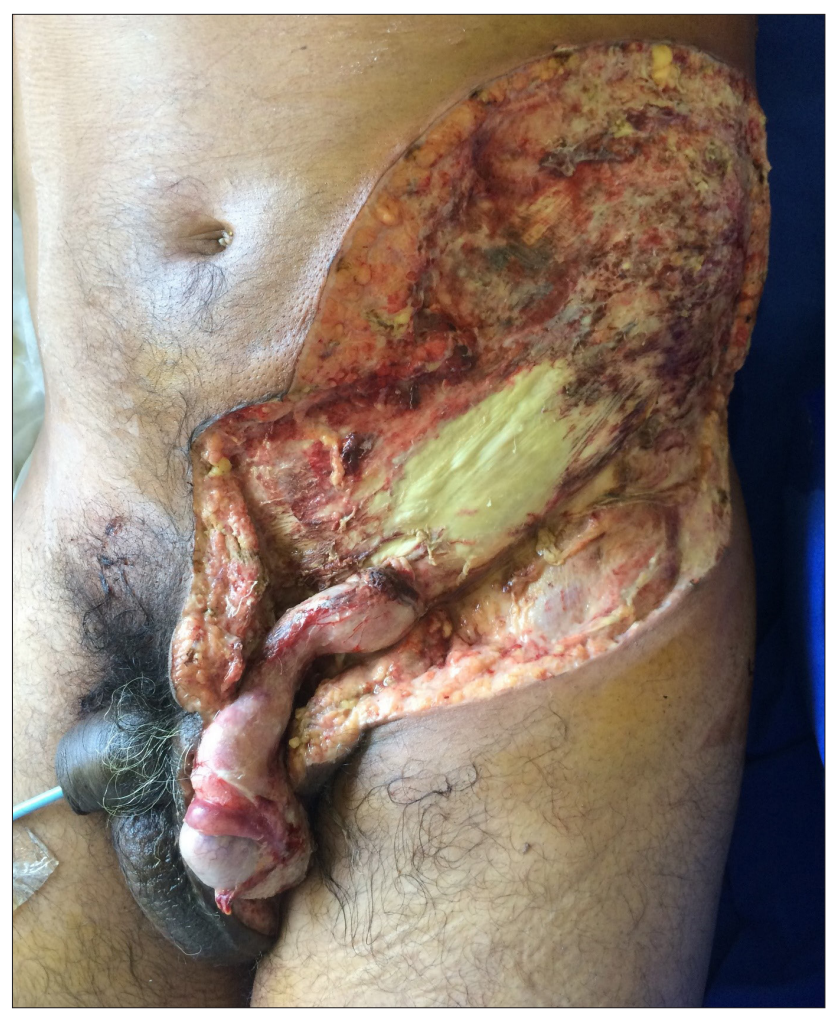

Fig. 3. A patient with extensive debridement of the left iliac fossa, immediately after debridement. debridement outside the perineum (onto the abdominal wall, chest or limbs) and requirement for organ support was present in $80.0 \%$ of the patients who died.

The parameters assessed as risk factors for mortality on univariate logistic regression are summarised in Table 4.

Of the parameters in Table 4, only requirement for organ support was a significant risk factor for mortality on multivariate analysis $(p=0.043)$. When FGSI $>9$, debridement outside the perineum and requirement for organ support were replaced in the multivariate regression by a single parameter that was positive only when these three conditions were met, this parameter was a significant risk factor for mortality $(p=0.002)$.

\section{Outcomes in HIV- and DM-affected patients}

There was a $14.3 \%$ mortality rate among HIV-positive patients and a $16.7 \%$ mortality rate among those with DM. Neither HIV $(p=0.658)$ nor DM $(p=0.386)$ was significantly associated with mortality. Patients with only DM were significantly more likely to need two or three debridements $(p=0.001)$, whereas the majority of patients without DM (92.3\%) required only one debridement. DM patients were significantly more likely to develop AKI ( $p=0.031$ ), but neither HIV nor DM was significantly associated with the need for organ support.

\section{Definitive management}

Most patients were managed at the tertiary centre with wound care, and ultimately went on to have skin grafts for coverage. Those with urethral strictures as the initiating event and those who went on to develop urethral strictures were planned for additional procedures to manage the strictures.

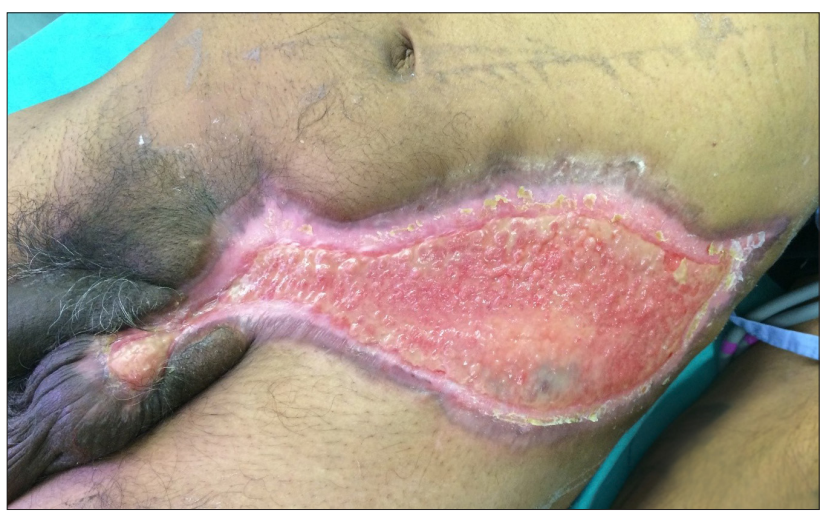

Fig. 4. The same patient as in Fig. 3 after wound care, prior to skin grafting.

Table 4. Parameters tested for association with mortality on univariate logistic regression

\begin{tabular}{|c|c|c|c|}
\hline Parameter & OR & 95\% CI for OR & $p$-value \\
\hline Age $>60$ years & 1.5 & $0.221-10.171$ & 0.678 \\
\hline $\mathrm{DM}$ & 2.4 & $0.358-16.077$ & 0.367 \\
\hline HIV & 1.75 & $0.263-11.662$ & 0.563 \\
\hline Lactate $\geq 3 \mathrm{mmol} / \mathrm{L}$ & 13.5 & $1.340-135.983$ & $0.027^{*}$ \\
\hline FGSI $>9$ & 16.8 & $1.526-184.921$ & $0.021^{*}$ \\
\hline Debridement outside perineum & 11.6 & $1.156-116.417$ & $0.037^{*}$ \\
\hline$\geq 2$ debridements & 4.35 & $0.633-29.914$ & 0.135 \\
\hline Requirement for organ support & 90 & $6.845-1183.325$ & $0.001^{*}$ \\
\hline FGSI $>9$, debridement outside perineum and requirement for organ support & 152 & $7.899-2924.764$ & $0.001^{*}$ \\
\hline
\end{tabular}




\section{Discussion}

In developing countries such as SA, poor socioeconomic conditions, limited access to healthcare, a high prevalence of HIV infection and an increasing prevalence of lifestyle diseases such as DM result in FG remaining an important clinical condition. It was not possible to calculate the incidence of FG in the present study, as Grey's Hospital is not the only hospital in the drainage area at which FG cases are treated. However, the caseload of 8.8 cases per year is certainly high. Sorensen and Krieger ${ }^{[12]}$ reviewed 1641 men with FG in the USA and observed that only $1 \%$ of hospitals in that country treated $>5$ cases of FG per year.

It has been well established that FG is associated with an underlying systemic condition that impairs host immunity. ${ }^{[3,4]}$ In the present study, HIV was the most common comorbidity, with $47.7 \%$ of patients being HIV-positive. This rate of HIV infection is higher than seen in other large studies. Ngugi et al. ${ }^{[13]}$ reviewed 146 patients presenting with FG in Kenya and found an HIV rate of $16.4 \%$. Chalya et al. ${ }^{[14]}$ reviewed 84 patients with FG in Tanzania and found an HIV rate of $11.3 \%$. SA has the highest prevalence of HIV in the world, so this finding is not surprising.

The mean age of presentation across all patients in the present study was 51 years. This is in line with other large studies. The mean age of the 1641 men with FG in Sorensen and Krieger's ${ }^{[12]}$ study was 50.9 years. In the present study, HIV-positive patients presented at a younger age (mean 41 years) than non-HIV positive patients. Ngugi et al.$^{[13]}$ found HIV to be the commonest underlying comorbidity in their Kenyan study, in which the patients had a mean age of 35 years. Elem and Ranjan ${ }^{[6]}$ reviewed 10 cases at a single hospital in Zambia and found $80 \%$ to be HIV-positive, with a median age of 34.5 years for the HIV-positive patients. These two studies did not compare the age of presentation in HIV-positive and HIV-negative patients, but still add credibility to the conclusion that HIV-positive patients are likely to present at a younger age.

All patients in the present study were initially treated with broadspectrum antibiotics. The most common choice was co-amoxiclav, metronidazole and gentamicin. FG is well known to be a synergistic polymicrobial infection, and generally not all organisms involved are identified in cultures. ${ }^{[3]}$ Broad-spectrum empirical antibiotic cover that includes Gram-positive, Gram-negative and anaerobic organisms is therefore necessary. The hospital protocol of co-amoxiclav, gentamicin and metronidazole seems to be reasonable empirical therapy for FG.

Although FG is rare, each case treated places a resource burden on the health system. The mean LoS in the present study was 26 days. In referral centres where bed occupancy rates are high, prolonged admissions increase bed pressure. Furthermore, patients with FG needed on average 1.33 surgical procedures each during their initial management, not considering additional procedures for skin grafting and management of complications. Nearly half of the patients were transfused, and $13.6 \%$ were identified as requiring ICU admission. These activities, along with other resources utilised, result in an inflated cost per admission in FG patients. Jiménez-Pacheco et al. ${ }^{[15]}$ investigated the cost of an FG admission in a Spanish study and found a cost of EUR25 108.67 per admission for a patient requiring ICU admission and at least one surgical procedure. They concluded that the cost was high and justified implementation of primary and secondary prevention measures.

The prolonged LoS in the present study is not unique, particularly compared with developing countries. Mean LoS was 28 days in a Zambian study, ${ }^{[6]} 37.1$ days in a Nigerian study, ${ }^{[16]} 23.2$ days for patients with complications in a Kenyan study ${ }^{[13]}$ and 28 days in a Tanzanian study. ${ }^{[14]}$ Mean LoS was even high (27.5 days) in a Spanish study ${ }^{[15]}$ In contrast, in Sorensen and Krieger's ${ }^{[12]}$ study in the USA, mean LoS was 10 days for teaching hospitals and 7 days for non-teaching hospitals. This difference is probably explained by far distances to referral centres and lack of capacity at district hospitals in developing countries, hindering downward referral.

FG is well known to be associated with a high risk of mortality. The overall mortality rate in the present study was $11.4 \%$, which is in line with other studies. Sorensen and Krieger ${ }^{[12]}$ analysed 59 FG case series published between 1972 and 2015 and found a mortality rate ranging between $0 \%$ and $88 \%$. In their review of 1641 men with FG in the USA, they found an overall case fatality rate of $7.5 \%$. Eke ${ }^{[17]}$ reviewed 1726 cases of FG in a meta-analysis and found a mortality rate of $16 \%$.

In the present study, neither HIV nor DM was significantly associated with increased mortality. This finding is surprising, given the immune compromise associated with these conditions. ${ }^{[3,4]} \mathrm{HIV}$ with a low $\mathrm{CD} 4+$ count was found to be significantly associated with increased mortality by Chalya et al. ${ }^{[14]}$ There was also no association between age and increased mortality, which is surprising, because older patients would be expected to cope less well with the physiological derangements associated with FG. Other studies have found that younger patients are more likely to survive than older patients. ${ }^{[2,15,18-20]}$ The likely explanation for these observations is that the small sample size of patients who died precluded a demonstrable correlation between mortality, comorbidity and age.

Owing to the frequent need for referral and the challenge of allocating resources in a resource-constrained environment, tools that enable outcome prediction are important in conditions such as FG. A number of these have been assessed, including the FGSI, Uludag FGSI (FGSI with age and extent of disease scores added), Age-adjusted Charlson Comorbidity Index (ACCI) and Surgical Apgar (sAPGAR) Score. ${ }^{[19]}$ The affected body surface area ${ }^{[21]}$ and the Laboratory Risk Indicator for Necrotising Fasciitis (LRINEC) ${ }^{[22]}$ have also been assessed. These tools can be used at the bedside to score patients and assist with decisions to refer to a higher centre, admit to ICU or withhold treatment. In the present study, the FGSI was automatically calculated using a formula in Microsoft Excel. This could easily be incorporated into a mobile phone app or website and made accessible to doctors to use when managing patients with FG.

Of the scoring tools available, the FGSI has been the most investigated. It was described by Laor et al. ${ }^{[2]}$ in 1995 and is based on the Acute Physiology and Chronic Health Evaluation II (APACHE II) score. The utility of the FGSI has been validated by a number of studies ${ }^{[23-29]}$ showing a correlation between FGSI and mortality, and a threshold of 9 as being sensitive and specific for predicting mortality. Roghmann et al. ${ }^{[19]}$ found that simpler scores such as the sAPGAR and ACCI were equivalent to the FGSI and UFGSI scores, ${ }^{[19]}$ but did not find the FGSI to be inferior. Other studies have shown that the FGSI is unable to predict disease severity and survival. ${ }^{[30-32]}$ Tuncel et al. ${ }^{[20]}$ concluded in a subsequent study that addition of age and involved surface area parameters was necessary to predict mortality.

In the present study, the percentage area involved was not recorded routinely in the patient notes. As a result, neither this parameter nor the UFGSI could be calculated. The mean FGSI was significantly different between survivors and non-survivors. An FGSI cut-off of 9 was significantly associated with mortality. Additional factors that were associated with mortality on univariate analysis were a lactate level of $>3 \mathrm{mmol} / \mathrm{L}$, debridement outside the perineum and requirement for organ support in the ICU. Debridement outside the perineum is an indicator of extensive disease, and our finding 
that it was a risk factor concurs with other studies showing that the percentage of body surface area affected is a predictor of mortality. ${ }^{[21]}$ The only risk factor that remained significant on multivariate analysis was requirement for organ support. The multivariate analysis was limited by the small sample size, particularly in the group of patients who died.

A combination parameter including FGSI $>9$, requirement for organ support and extension outside the perineum was a significant risk factor for mortality. Based on validations of the FGSI in the literature and the findings of the present study, it appears that a scoring system for decision-making in FG must incorporate acute physiological derangement, extent of body surface area involvement and organ failure. The present study validates the FGSI in the SA population. It also supports the concept of adding additional parameters to the FGSI to better predict mortality, in this case presence of organ failure and disease extent. In the resourceconstrained SA environment, use of such a scoring system is likely to be useful in ICU bed and emergency theatre allocation when there are often multiple patients competing for a single resource, and patients with a high risk of mortality could potentially be offered palliative care.

\section{Conclusions}

FG remains a condition with a high mortality rate, and the caseload at an SA tertiary referral centre is high. HIV-positive patients present at a younger age but do not have worse outcomes. Patients with DM also do not have worse outcomes, but require more debridements and are more likely to develop AKI. In a resource-constrained environment, outcome prediction is necessary to enable resource allocation. The FGSI successfully predicts increased mortality using a threshold of 9. Patients with an FGSI $>9$ have a high risk of mortality and will benefit from ICU care. The combination of FGSI $>9$, requirement for organ support and extension beyond the perineum (onto the abdominal wall, chest or limbs) is associated with a very high risk of mortality and may be useful as an exclusion criterion when allocating scarce resources.

\footnotetext{
Acknowledgements. None.

Author contributions. All three authors conceptualised and planned the paper. AEE collected and organised the data. RJU reviewed the data and assisted with analysis of the data. AEE prepared the first draft of the article, RJU revised the article, and SM was a research supervisor and checked the final draft.

Funding. This study was supported by an educational grant from the South African Urological Association.

Conflicts of interest. None.

1. Thwaini A, Khan A, Malik A, et al. Fournier's gangrene and its emergency management. Postgrad Med J 2006;82(970):516-519. https://doi.org/10.1136/pgmj.2005.042069

2. Laor E, Palmer LS, Tolia BM, Reid RE, Winter HI. Outcome prediction in patients with Fournier's 2. Laor E, Palmer LS, Tolia BM, Reid RE, Winter HI. Outcome prediction in patients
gangrene. J Urol 1995;154(1):89-92. https://doi.org/10.1016/S0022-5347(01)67236-7

3. Shyam DC, Rapsang AG. Fournier's gangrene. Surgeon 2013;11(5):222-232. https://doi.org/10.1016/j. 3. Shyam DC, Rapsa
surge.2013.02.001
}

4. Singh A, Ahmed K, Aydin A, Khan MS, Dasgupta P. Fournier's gangrene: A clinical review. Arch Ital Urol Androl 2016;88(3):157-164. https://doi.org/10.4081/aiua.2016.3.157

5. Joint United Nations Programme on HIV/AIDS (UNAIDS). AIDSinfo. 2013. http://www.unaids.org/ en/ (accessed 3 January 2015).

6. Elem B, Ranjan P. Impact of immunodeficiency virus (HIV) on Fournier's gangrene: Observations in Elem B, Ranjan P. Impact of immunodeficiency virus
Zambia. Ann R Coll Surg Engl 1995;77(5):283-286.

Zambia. Ann R Coll Surg Engl 1995;77(5):283-286.
7. Johnin K, Nakatoh M, Kadowaki T, Kushima M, Koizumi S, Okada Y. Fournier's gangrene caused by Candida species as the primary organism. Urology 2000;56(1):153. https://doi.org/10.1016/S0090295(00)00527-6

8. Tleyjeh IM, Routh J, Qutub MO, Lischer G, Liang KV, Baddour LM. Lactobacillus gasseri causing Fournier's gangrene. Scand J Infect Dis 2004;36(6-7):501-503. https://doi. org $/ 10.1080 / 00365540410015916$

9. Sroczyński M, Sebastian M, Rudnicki J, Sebastian A, Agrawal AK. A complex approach to the treatment of Fournier's gangrene. Adv Clin Exp Med 2013;22(1):131-135.

10. Saijo S, Kuramoto Y, Yoshinari M, Tagami H. Extremely extended Fournier's gangrene. Dermatologica 1990;181(3):228-232. https://doi.org/10.1159/000247930

11. Chawla SN, Gallop C, Mydlo JH. Fournier's gangrene: An analysis of repeated surgical debridement. Eur Urol 2003;43(6):572-575. https://doi.org/10.1016/S0302-2838(03)00102-7

12. Sorensen MD, Krieger JN. Fournier's gangrene: Epidemiology and outcomes in the general US Sorensen MD, Krieger JN. Fournier's gangrene: Epidemiology and outco
population. Urol Int 2016;97(3):249-259. https://doi.org/10.1159/000445695

13. Ngugi P, Magoha G, Nyaga P. Fournier's gangrene in the HIV era. Afr Health Sci 2014;14(5):1063-1068. https://doi.org/10.4314/ahs.v14i4.38

14. Chalya PL, Igenge JZ, Mabula JB, Simbila S. Fournier's gangrene at a tertiary health facility in northwestern Tanzania: A single centre experiences with 84 patients. BMC Res Notes 2015;8:481. https://doi.org/10.1186/s13104-015-1493-1

15. Jiménez-Pacheco A, Arrabal-Polo MÁ, Arias-Santiago S, Arrabal-Martín M, Nogueras-Ocaña M, Zuluaga-Gómez A. Fournier gangrene: Description of 37 cases and analysis of associated health care costs. Actas Dermosifiliogr 2012;103(1):29-35. https://doi.org/10.1016/j.adengl.2011.04.007

16. Ugwumba FO, Nnabugwu II, Ozoemena OF. Fournier's gangrene - analysis of management and outcome in south-eastern Nigeria. S Afr J Surg 2012;50(1):16-19.

17. Eke N. Fournier's gangrene: A review of 1726 cases. Br J Surg 2000;87(7):718-728. https://doi. org/10.1046/j.1365-2168.2000.01497.x

18. Hsu JM, Chen M, Weng CH, Tseng JS. Fournier's gangrene: Clinical characteristics in the elderly. Int J Gerontol 2014;8(3):162-165. https:///doi.org/10.1016/i.ijge.2013.08.013

19. Roghmann F, von Bodman C, Löppenberg B, Hinkel A, Palisaar J, Noldus J. Is there a need for the Roghmann F, von Bodman C, Löppenberg B, Hinkel A, Palisaar J, Noldus J. Is there a need for the
Fournier's gangrene severity index? Comparison of scoring systems for outcome prediction in patients with Fournier's gangrene. BJU Int 2012;110(9):1359-1365. https://doi.org/10.1111/j.1464410X.2012.11082.X

20. Tuncel A, Keten T, Aslan Y, et al. Comparison of different scoring systems for outcome prediction in patients with Fournier's gangrene: Experience with 50 patients. Scand J Urol 2014;48(4):393-399. https://doi.org/10.3109/21681805.2014.886289

21. Morais H, Neves J, Maciel Ribeiro H, et al. Case series of Fournier's gangrene: Affected body surface area - the underestimated prognostic factor. Ann Med Surg 2017;16:19-22. https://doi.org/10.1016/j. amsu.2017.02.043

22. Wong CH, Khin LW, Heng KS, Tan KC, Low CO. The LRINEC (Laboratory Risk Indicator for Necrotizing Fasciitis) score: A tool for distinguishing necrotizing fasciitis from other soft tissue infections. Crit Care Med 2004;32(7):1535-1541. https://doi.org/10.1097/01.CCM.0000129486.35458.7D

23. Yeniyol CO, Suelozgen T, Arslan M, Ayder AR. Fournier's gangrene: Experience with 25 patients and use of Fournier's gangrene severity index score. Urology 2004;64(2):218-222. https://doi.org/10.1016/j. urology.2004.03.049

24. Corcoran AT, Smaldone MC, Gibbons EP, Walsh TJ, Davies BJ. Validation of the Fournier's gangrene severity index in a large contemporary series. J Urol 2008;180(3):944-948. https://doi.org/10.1016/j. juro.2008.05.021

25. Uluğ M, Gedik E, Girgin S, Celen MK, Ayaz C. The evaluation of microbiology and Fournier's gangrene severity index in 27 patients. Int J Infect Dis 2009;13(6):e424-e430. https://doi.org/10.1016/j. id.2009.01.021

26. Unalp HR, Kamer E, Derici H, et al. Fournier's gangrene: Evaluation of 68 patients and analysis of prognostic variables. J Postgrad Med 2008;54(2):102-105. https://doi.org/10.4103/0022-3859.40775

27. Lin $\mathrm{E}$, Yang $\mathrm{S}$, Chiu $\mathrm{AW}$, et al. Is Fournier's gangrene severity index useful for predicting outcome of Fournier's gangrene? Urol Int 2005;75(2):119-122. https://doi.org/10.1159/000087164

28. Morua AG, Lopez JA, Garcia JD, Montelongo RM, Guerra LS. Fournier's gangrene: Our experience in 5 years, bibliographic review and assessment of the Fournier's gangrene severity index. Arch Esp Urol 2009;62(7):532-540.

29. Tarchouli M, Bounaim A, Essarghini M, et al. Analysis of prognostic factors affecting mortality in Fournier's gangrene: A study of 72 cases. Can Urol Assoc J 2015;9(11-12):E800-E804. https://doi. org $/ 10.5489 /$ cuaj.3192

30. Tuncel A, Aydin O, Tekdogan U, Nalcacioglu V, Capar Y, Atan A. Fournier's gangrene: Three years of experience with 20 patients and validity of the Fournier's Gangrene Severity Index Score. Eur Urol
entris experience with 20 patients and validity of the Fournier's Gang

31. Janane A, Hajji F, Ismail TO, et al. Hyperbaric oxygen therapy adjunctive to surgical debridement in management of Fournier's gangrene: Usefulness of a severity index score in predicting disease gravity and patient survival. Actas Urol Esp 2011;35(6):332-338. https://doi.org/10.1016/j.acuro.2011.01.019

32. Luján Marco S, Budía A, Di Capua C, Broseta E, Jiménez Cruz F. Evaluation of a severity score to predict the prognosis of Fournier's gangrene. BJU Int 2010;106(3):373-376. https://doi.org/10.1111/ 1.1464-410X.2009.09075.x

Accepted 2 February 2018. 